A N N A L E S Annales de Bretagne et des Pays de l'Ouest

Anjou. Maine. Poitou-Charente. Touraine

$110-3 \mid 2003$

Varia

\title{
Pádraig Ó Riain, Four Irish Martyrologies Four Irish Martyrologies
}

\section{Bernard Merdrignac}

\section{(2) OpenEdition}

1 Journals

Édition électronique

URL : http://journals.openedition.org/abpo/1397

DOI : $10.4000 /$ abpo. 1397

ISBN : 978-2-7535-1491-1

ISSN : 2108-6443

Éditeur

Presses universitaires de Rennes

Édition imprimée

Date de publication : 20 octobre 2003

Pagination : 135-137

ISBN : 978-2-86847-929-7

ISSN : 0399-0826

\section{Référence électronique}

Bernard Merdrignac, «Pádraig Ó Riain, Four Irish Martyrologies Four Irish Martyrologies », Annales de Bretagne et des Pays de l'Ouest [En ligne], 110-3 | 2003, mis en ligne le 20 octobre 2005, consulté le 23 septembre 2020. URL : http://journals.openedition.org/abpo/1397 ; DOI : https://doi.org/10.4000/ abpo. 1397 


\section{Comptes rendus}

Ó RIAIN, Pádraig, Four Irish Martyrologies : Drummond, Turin, Cashel, York, Henry Bradshaw Society, vol. 115, London, 2002, $260+$ XIV p., £ 35, \$ 60 .

Sans doute par suite des aléas de la conservation, l'Irlande médiévale nous a transmis fort peu de manuscrits liturgiques médiévaux (et notamment de bréviaires et de missels) en comparaison de la Grande Bretagne ou du Continent. Par contre, les martyrologes (et les calendriers liturgiques versifiés en langue vernaculaire : féliri) constituent les principaux documents d'histoire religieuse émanant de l'" île des saints et des savants ", ce qui confirme l'importance du culte des saints dans l'Église irlandaise. Toutefois, la tradition manuscrite est fort complexe étant donné les vicissitudes de la conjoncture historique. Pour ne retenir que deux exemples, le plus ancien manuscrit (Leabar Brecc) comportant le "Martyrologe d'Oengus le culdée " (Félire Oengusso) dont la composition remonte aux environs de 830 n'est pas antérieur au début du $\mathrm{XV}^{\mathrm{e}}$ siècle; le "Martyrologe de Gorman " (Félire húi Gormáin : fin XII e siècle) n'est plus accessible que dans une copie réalisée en 1630 par Michael Ó Cleary qui participait à l'entreprise de collectage hagiographique orchestrée par les franciscains irlandais du collège Saint-Antoine de Louvain.

Le professeur Pádraig Ó Riain qui fait autorité dans le domaine de l'hagiographie irlandaise a édité ici quatre martyrologes qui se situent dans la tradition du Félire Oengusso. Ce travail, éditorial entrepris voici une quinzaine d'années, mais différé, de l'aveu de l'auteur, par suite de tâches plus urgentes (dont les $A B P O$ se sont à l'occasion fait l'écho), s'inscrit ainsi dans la ligne de la Henry Bradshaw Society pour qui "l'édition de textes liturgiques rares" est l'un des objectifs primordiaux depuis sa fondation. Au départ, il s'agissait pour l'éditeur de mettre à la disposition des chercheurs deux matyrologes d'origine irlandaise récemment découverts dans un manuscrit (D IV 18) de la Biblioteca Nazionale de Turin. Mais la nécessité s'est imposée d'adjoindre à l'ouvrage deux autres documents importants. Le " Martyrologe de Drummond " n'avait pas été réédité depuis la publication à la fin du XIX ${ }^{\mathrm{e}}$ siècle par G. H. Forbes de The Ancient Irish Missal in the Possession of the Baroness Willoughby de Eresby, Drummond Castle, Perthshire, Edinburg, 1882 (ouvrage devenu inaccessible et obsolète). Entretemps, ce précieux manuscrit à été acquis (en 1916) par la Pierpont Morgan Library (New York). Quant au " Martyrologe de Cashel ", il n'avait jamais été édité jusqu'à présent; et pour cause, puisque le manuscrit a disparu depuis le XvII $^{\mathrm{e}}$ siècle où le franciscain John Colgan l'a mis à contribution dans les notes de ses deux ouvrages fondamentaux, les Acta Sanctorum Hiberniae et les Tria Thaumaturga (1645-1647), issus des recherches des franciscains irlandais. C'est ce qui explique que le texte reconstitué ici par P. Ó Riain soit en latin, alors que l'original devait être en irlandais (voir p. 162 n. 2). Le présent compte rendu ne 
saurait prétendre aborder tous les champs de recherche dans lesquels l'apport de ce type de sources est susceptible d'enrichir la réflexion des historiens des mentalités religieuses médiévales. Une seule remarque (p. 166, n. 23) suffira pour jauger la profondeur de l'inculturation dont étaient susceptibles les liturgistes irlandais du Moyen Âge. Le " Martyrologe de Cashel " célèbre, le 14 juin, saint Benoît présenté comme le successeur (" comorbanus " : comarba = " héritier ") de saint Enda d'Aran et le frère de saint Ciaran de Saighir! On sait que, dans le même esprit, certaines traditions insulaires (dans tous les sens du terme!) n'ont pas hésité à naturaliser saint Grégoire le Grand ni à faire de sainte Brigitte la "Marie des Gaels "...

On se contentera donc ici de faire brièvement ressortir l'intérêt de la démarche de Pádraig Ó Riain - avec l'acribie qui constitue en quelque sorte la marque de fabrique de ce chercheur - pour situer le plus précisément possible ces quatre textes dans leur contexte de rédaction. Le Martyrologe de Drummond présente la particularité d'avoir sans doute été conservé dans le manuscrit original. À la suite d'une discussion serrée, l'éditeur établit que ce martyrologe a été composé à Armagh, peu avant la conquête de l'Irlande (1169) par les Plantagenêts (la date de 1174 constituant un terminus ad quem) à destination de Glendalough. On y décèle l'influence des prescriptions du synode de Clane (1162) qui enjoint aux Églises d'Irlande "règle et bonne conduite " (ce qui implique l'établissement d'ouvrages liturgiques corrects). Dans ces conditions, il y a de bonnes raisons de d'attribuer la rédaction de ce martyrologe à Flann Ua Gormáin († 1174), " archi-lecteur " de l'école d'Armagh, connu par ailleurs pour être l'auteur d'un commentaire du Félire Oengusso, et frère de Máel Muire Ua Gormáin, auteur du Félire húi Gormáin. Un argument emporte la conviction et rend compte de la minutie de l'enquête menée par P. Ó Riain. En effet les folii 107-108 du manuscrit révèlent l'intervention d'une " main non-irlandaise " que les paléographes considèrent comme de peu antérieure au milieu du XII ${ }^{\mathrm{e}}$ siècle. Or Flann est censé avoir passé une vingtaine d'années chez les «Francs et les Saxons " avant son retour à Armagh en 1154. Il est donc permis de conjecturer que ce serait durant ce séjour à l'étranger que ce scribe aurait acquis la maîtrise de l'écriture qui distingue ces folii des autres.

Comme le Félire húi Gormáin (1168-1170) est la principale source des gloses au Félire Oengusso pour lesquelles la date du décès de Flann Ua Gormáin en 1174 constitue un terminus ante quem, le "Martyrologe de Turin " qui s'inspire étroitement de celles-ci ne peut être antérieur au dernier quart du xII ${ }^{\mathrm{e}}$ siècle. La critique interne de ce document (qui a été relié - probablement au XIV ${ }^{\mathrm{e}}$ siècle - avec un manuscrit des Homélies sur les Évangiles de Grégoire le Grand) conduit l'éditeur à en attribuer la confection à l'usage du couvent de religieuses augustiniennes de Skreen (peut-être fondé dès 1144). Or cet établissement a été remplacé vers 1240 par le couvent de Lismullin ( ${ }^{*}$ Les Moling, dédié à saint Moling Luachra) implanté à proximité sous les auspices du conquérant anglais. Le "Martyrologe de Turin" (dont les termini s'inscrivent donc entre 1174 et 1240) serait ainsi le plus ancien manuscrit conservé à avoir été composé à destination d'un couvent de religieuses irlandaises (p. 130).

Sans revenir sur la procédure de reconstitution du " Martyrologe de Cashel " à partir des annotations des ouvrages de John Colgan et de quelques notes manuscrites de cet auteur conservées à la Franciscan Library de Killiney, notons que ce document procède aussi d'une version glosée du Félire Oengusso, ce qui implique encore dans ce cas une datation qui ne peut être antérieure au dernier quart du XII ${ }^{\mathrm{e}}$ siècle. La teneur du texte atteste d'un intérêt particulier pour le diocèse de Lismore dont il pourrait bien provenir, plutôt que de celui de Cashel, 
comme l'affirmait Colgan. Enfin, le manuscrit D IV 18 de la Biblioteca Nazionale de Turin comporte dans les marges inférieures de ses premiers folii des extraits d'une version d'origine irlandaise d'un Martyrologe versifié (connu par ailleurs par un manuscrit rémois du $\mathrm{IX}^{\mathrm{e}}$ siècle, publié au XVII ${ }^{\mathrm{e}}$ siècle par Luc d'Achery et disparu depuis). Ce dernier aurait été compilé soit pour l'Église d'York, soit pour celle de Ripon et Michel Lapidge estime qu'il pourrait remonter à la seconde moitié du VIII ${ }^{\text {e }}$ siècle. Quant à la version irlandaise (en hexamètres dactyliques irréguliers comme sa source originelle), sous réserve d'études ultérieures, celleci a pu être réalisée durant une large fourchette chronologique, du $\mathrm{VIII}^{\mathrm{e}}$ siècle au $\mathrm{XII}^{\mathrm{e}}$ siècle, et elle provient probablement de Clonmacnoise. En tout cas, sa présence dans le manuscrit de Turin atteste qu'elle était en usage dans l'Église irlandaise à la fin du XII ${ }^{\mathrm{e}}$ siècle.

L'érudition déployée dans cet ouvrage par le professeur Pádraig Ó Riain procure au lecteur une incontestable satisfaction intellectuelle. Toutefois ce plaisir n'est pas gratuit. En replaçant ces productions liturgiques dans leur contexte historique, cette publication impeccable des derniers Martyrologes irlandais demeurés inédits (ou du moins devenus difficiles d'accès) permet de mieux appréhender l'action des chanoines augustiniens qui tissaient leur réseau de prieurés à un tournant de l'histoire de l'Église irlandaise, au moment de l'intervention dans l'île des Plantagenêts et de leurs vassaux.

Bernard MERDRIGNAC

Merdrignac, Bernard, Le sport au Moyen Âge, Rennes, Presses universitaires de Rennes, 2002, 340 p., $13 €$.

Sous un titre relativement provoquant, Bernard Merdrignac nous offre dans un livre très dense moins une histoire du sport au Moyen Âge qu'une réflexion sur ce que pouvait être la relation des sociétés médiévales avec l'activité physique et le corps, le jeu et le loisir. La mise en perspective de toute une série d'activités bien médiévales comme les tournois, des rapprochements parfois surprenants mais révélateurs offrent beaucoup plus qu'un manuel commode, ce que peut-être aussi cet ouvrage, fruit d'un enseignement auprès d'étudiants "sportifs ". Écrit d'un style alerte et non sans un humour, relayé par un caricaturiste débutant, cet essai propose aussi une foule de renseignements précis et divers qui couvrent nombre d'activités physiques dans un espace géographique des plus vastes où apparaît fréquemment l'Irlande chère à l'auteur et passablement méconnue. Des compléments bibliographiques, un glossaire, des notices biographiques et surtout un abondant dossier de soixante-dix pages de documents souvent très originaux complètent fort bien l'ouvrage.

Le premier chapitre invite à une réflexion nécessaire. Le sport moderne est né au XIX ${ }^{\mathrm{e}}$ siècle dans le cadre d'une société bien spécifique mais ne peut-on pas parler, d'une certaine manière, de sport dans les périodes antérieures? L'auteur fait alors appel aux théoriciens qui ont proposé les lectures les plus diverses, voire les plus opposées. Le sport est-il inhérent à la nature humaine et, dans ce cas, il n'aurait guère d'histoire, se cantonne t-il dans une naissance contemporaine, est-il le fruit d'une évolution d'une "sportivisation " du jeu, le fruit d'un "processus de civilisation " comme le propose Norbert Hélias? Pour aborder le sujet et rechercher les aspects sportifs dans nombre d'activités médiévales, B. Merdrignac s'appuie largement sur la théorie exposée par R. Caillois dans Les 
jeux et les hommes, faisant du jeu une activité " séparée, incertaine, improductive, réglée et fictive ». Si le Moyen Âge a ignoré le sport, il a connu un concept particulier qui semble y préluder en partie sous le nom de déport qui s'applique au divertissement qui peut se faire dans le cadre de compétitions désignées par le mot ébattement.

Ces bases posées, il est indispensable d'examiner la position des médiévaux à l'égard du corps. Ici, B. Merdrignac fait justice de nombreux clichés en montrant que le Moyen Âge n'a pas eu systématiquement le mépris du corps qu'on lui prête souvent et que la position demeura beaucoup plus nuancée, la règle de saint Benoît se montre particulièrement équilibrée à cet égard. L'étude de l'éducation physique au Moyen Âge le montre bien. Les jeux sportifs sont multiples, de la lutte au lancer de pierre, et l'étude des récréations enfantines dévoile d'autres exemples. Une place particulière est consacrée à la formation physique du chevalier. L'étude des enfances de Duguesclin et de Boucicault est très révélatrice mais, si cela prend pour nous bien des aspects sportifs, l'inspiration générale est tout autre.

Les chapitres suivants sont consacrés à l'analyse d'activités qui présentent un grand nombre de caractères sportifs et entretiennent des liens apparents avec des sports modernes. La chasse à courre ou au faucon apparaît très vite comme un loisir fortement aristocratique, un véritable marqueur social et l'auteur rappelle avec à propos que le véritable sportsman anglais fut d'abord l'aristocrate qui se livrait à la chasse au renard. Joutes et tournois, et les jeux guerriers en général, présentent beaucoup d'aspects sportifs : spécialité aristocratique, engagement physique, compétition et spectacle.

Les jeux de balle faillirent devenir un vrai sport et la paume n'est pas étrangère au tennis moderne. $B$. Merdrignac tente de démêler l'écheveau complexe des continuités qui n'en sont pas, des ruptures et des implications sociales. D'abord apanage des clercs, le jeu de balle se popularisa et gagna de larges couches de la population. Par bien des traits, le sport véritable se dessine : le terrain propre dérivé du cloître où jouaient les clercs, l'élaboration de règles etc. Mais l'association dans les esprits de la paume à la France ancienne ruina son crédit. Pareillement, la soule, pratique populaire violente, peut présenter des analogies avec foot-ball et rugby mais elle s'inscrit profondément dans l'identité villageoise et demeura un jeu peu réglementé.

Tout en restant très ouvert, le livre propose une mise en perspective des activités physiques en les reliant profondément aux pratiques sociales du Moyen Âge et provoque une réflexion nourrie qui lance des perspectives éventuellement inattendues sur des réalités classiques du Moyen Âge. Si l'on ajoute l'intérêt du gros dossier documentaire qui nous offre des textes souvent rares, l'ensemble se présente comme un livre riche et stimulant, dommage que des coquilles un peu abondantes le déparent quelque peu.

Daniel PICHOT

Pichot, Daniel, Le village éclaté. Habitat et société dans les campagnes de l'Ouest au Moyen Âge, PUR, 2002, 395 p.

L'ouvrage de Daniel Pichot, fruit de son habilitation, est d'abord et avant tout une réflexion sur la définition et, ce faisant, la naissance du village dans cette France de l'Ouest (Anjou, Maine et Bretagne) profondément marquée par 
l'habitat dispersé. L'entreprise est ambitieuse et courageuse face aux lacunes documentaires et aux controverses ambiantes. Historiens et archéologues sont loin d'être unanimes et peinent à cerner le phénomène tant en France qu'ailleurs. Entre ce que d'aucuns appellent les " proto-villages " du haut Moyen Âge et les villages des $\mathrm{XI}^{\mathrm{e}}$-XII ${ }^{\mathrm{e}}$ siècles nés de l'encellulement (R. Fossier) et de l'incastellamento (P. Toubert), les perspectives enrichies de nouveaux apports, archéologiques notamment, connaissent un notable renouvellement dont témoignent toute une série de travaux récents.

Le titre, agréablement provocateur - "le village éclaté »! -, résume à la perfection les conclusions de l'auteur. Il y a un processus notable de regroupement aux Xle et XII ${ }^{\mathrm{e}}$ siècles mais il est incomplet et laisse, aux niveaux des impulsions motrices, la part belle à l'église dans le cadre de la paroisse (noyaux ecclésiaux...). L'image qui se dégage est peu conforme au modèle de l'habitat groupé. La dispersion, aux formes diversifiées, règne en maîtresse mais il y a bien en arrière-plan un finage organisé et une communauté agissante de villageois. Le village est éclaté.

On saura gré à Daniel Pichot d'avoir rassemblé une telle masse d'informations et dressé un état de la recherche qui intègre des données multiformes issues tant de la documentation écrite que des apports archéologiques (cadastres, prospections, fouilles...). Le propos est ferme et bien construit. Après un long examen critique des sources, l'auteur s'interroge sur la période de gestation (haut Moyen Âge) avant de se consacrer aux temps forts de son étude, les $\mathrm{XI}^{\mathrm{e}}$-XII ${ }^{\mathrm{e}}$ siècles et dans une moindre mesure le XIII ${ }^{\mathrm{e}}$ siècle. Sont successivement examinés les pôles de cristallisation, la typologie des habitats (villages, hameaux, habitats isolés), les finages puis l'encadrement paroissial et seigneurial et enfin la société villageoise. L'auteur insiste prudemment. Sur un aussi vaste territoire, l'enquête ne saurait être ni exhaustive, ni définitive. Son but est de proposer un modèle explicatif propre à nourrir la réflexion et à susciter de nouvelles recherches.

Au niveau méthodologique, les archéologues ne peuvent être que séduits par un travail qui intègre leurs recherches, mais rappelons toutefois que l'on ne dispose en la matière que d'un corpus documentaire d'une grande indigence, d'où ces citations à répétition des - voire du - mêmes articles qui laissent un peu perplexe. Quant à l'emploi des cadastres " napoléoniens ", s'il est parfaitement légitime et en l'occurrence mené ici avec mesure et circonspection, insistons sur le fait que dans ce domaine on ne l'est jamais assez, tant il est difficile de retrouver sous les formes du XIX ${ }^{\mathrm{e}}$ siècle les réalités des $\mathrm{XI}^{\mathrm{e}}$-XIII ${ }^{\mathrm{e}}$ siècles. Pour ce qui est des conclusions du volume, je serais personnellement - au vu des exemples manceaux - moins encline que Daniel Pichot à réduire la part de l'incastellamento auquel on doit localement tout le réseau des petites villes - ces anciens villages dont la création n'a pu que sérieusement influer, que ce soit directement ou indirectement, sur la mise en place et les caractéristiques du peuplement rural au sein du comté du Maine. Sur la forme, j'exprimerai un regret. Il concerne les figures qui pour nombre d'entre sont un peu floues et auraient mérité un autre format. Mais tout ceci n'est que peu de chose.

Ce bel ouvrage particulièrement érudit, minutieux et prudent est un ouvrage de référence qu'historiens et archéologues, tant ceux de l'Ouest que d'ailleurs, devront méditer et tester à l'aune des enquêtes locales. 
Hommage à Arthur de La Borderie. Études, documents et actes du colloque, Bulletin et mémoires de la Société Archéologique et Historique d'Ille-et-Vilaine, t. CVI, 2002, 318 p. (volume en vente au siège de la société, 3 rue Yves-Mayeuc, 35000 Rennes, $30 €)$.

La société Archéologique et Historique d'Ille-et-Vilaine ne pouvait manquer de commémorer le centenaire de la mort de son fondateur et président pendant de longues années. Par ailleurs, revisiter ce monument de l'histoire bretonne ne manquait pas d'intérêt. Ce volume contient surtout le colloque réuni en 2001 à Rennes et y sont jointes quelques contributions qui viennent éclairer le personnage. Pour être nécessaire, l'entreprise n'allait pas sans danger. Le résultat montre que les écueils ont été évités : l'apologie souvent de mise pour ce genre de manifestation aussi bien qu'une remise en cause d'une œuvre historique souvent bien dépassée. Le lecteur y trouvera donc de multiples attraits.

En premier lieu, apparaît évidemment l'historien. Face à une œuvre impressionnante qui réunit nombre de publications de textes, des articles multiples et, bien sûr, la monumentale Histoire de Bretagne, les historiens d'aujourd'hui rendent hommage au grand maître mais affirment des positions critiques, tant sur la méthode que les conclusions. La Borderie a commencé très jeune son travail de recherche, sa méthode, ses idées de base se sont fixées tôt dans le $\mathrm{XIX}^{\mathrm{e}}$ siècle et il appartient sans doute plus à la génération romantique qu'à celle des historiens critiques, beaucoup plus rigoureux de la fin du XIX ${ }^{\mathrm{e}}$ siècle. Aujourd'hui les historiens ne peuvent que pointer ses faiblesses : méconnaissance de certaines sources, confiance excessive dans la valeur historique des vitae, bref une méthode qui ne peut satisfaire comme le disent $\mathrm{H}$. Guillotel et J. Quaghebeur, l'un en examinant l'attitude de La Borderie envers les sources, l'autre en se référant à l'histoire de la Cornouaille. J.-C. Cassard, quant à lui, examine la démarche dans l'histoire de la guerre de succession de Bretagne où La Borderie suit assez servilement les chroniques de Froissart qui n'est qu'assez peu fiable. De façon générale, les intervenants lui reprochent de travailler vite, entrant en concurrence parfois avec des confrères dans la publication des vies de saints et E. Guitton résume le propos en écrivant : "La Borderie a fait un travail utile, non un travail exemplaire. Ce chartiste n'était point dénué d'amateurisme, sur bien des points il se contentait d'à-peu-près. » Le jugement est sévère mais, comme le souligne B. Merdrignac dans la table ronde finale, ses éditions imparfaites ont le mérite d'exister et rendent encore d'excellents services, en particulier, la Sociétés des Bibliophiles bretons qu'il a fondée a mis à la disposition des chercheurs nombre de documents de grande importance.

Plusieurs contributions viennent ensuite apporter des éclairages sur l'engagement de La Borderie dans l'animation de la recherche et la défense du patrimoine. M. Duval décrit son rôle dans la fondation de la section d'Histoire et D'Archéologie de l'Association bretonne. Il montre un tout jeune homme de moins de 20 ans animant le débat par des interventions originales. Plus tard, reconnu par ses pairs, il assume un rôle non négligeable dans la protection du patrimoine. Il s'intéresse aux travaux de restauration du chanoine Brune dans les églises du département d'llle-et-Vilaine et intervient activement à l'occasion pour sauver un édifice menacé. Il ne remporta pas que des succès mais l'église de Saint-Lunaire lui doit de subsister, cela n'empêcha pas pourtant de nombreuses destructions.

Enfin, M. Denis brosse une étude largement ouverte sur des comparaisons avec toute l'Europe sur le rôle d'inspirateur du nationalisme breton joué par l'historien. S'il n'est pas sans points communs avec les intellectuels à l'origine de nombreux mouvements qui, à son époque, secouaient les peuples d'Europe 
centrale, il s'arrêta à la première étape en exaltant une nation bretonne idéale mais il ne fonda pas d'organisation et ne milita pas, d'abord parce que pour lui la nation bretonne s'était évanouie et appartenait au passé. Il se voulait chantre de cette patrie bretonne et totalement fidèle à la grande patrie, la France. On le comprend, les mouvements nationalistes ne purent en faire vraiment leur héraut et se trouvaient en porte à faux face à sa pensée.

Les communications font le point sur les travaux et l'action de La Borderie et c'est déjà beaucoup, même si les avis divergent quelque peu, mais on peut trouver plus dans ce livre : un homme, un véritable intellectuel d'une ampleur indiscutable et donc l'action multiforme imprima sa marque à son époque. Un portrait émane des différents articles, à commencer par ceux qui sont explicitement centrés sur l'aspect biographique, la grande synthèse initiale de J. Gury ou celui que $\mathrm{X}$. Ferrieu consacre à sa jeunesse et à ses années de formation. La Borderie apparaît comme profondément inscrit dans son siècle. C'est un bourgeois aisé, capable de vivre de ses rentes et de se consacrer à ses études en toute liberté. C'est aussi un conservateur, monarchiste mais relativement modéré. En notable, il abordera la carrière politique qui le mènera à la députation au début de la III ${ }^{\mathrm{e}}$ République mais sans grande passion. Par contre, il se révèle un animateur hors pair qui fonde et anime associations et sociétés savantes, qui mène le débat dans de multiples congrès et atteint une position dominante dans le travail historique en Bretagne. Enfin, au fil des pages se dévoile ce qu'était l'histoire selon lui et c'est à une passionnante recherche d'historiographie que nous sommes conviés. Ainsi revit, une aventure intellectuelle, une étape de la recherche historique et l'on peut regretter que l'historien ne soit jugé que par rapport à notre temps, ce qui lui vaut évidemment de graves reproches mais il n'est évoqué que trop occasionnellement ce qu'il apporta par rapport à ses prédecesseurs et qui ne fut pas mince.

Au-delà du bilan, indispensable bien sûr, de l'historien ce volume nous apporte le portrait très fouillé d'un intellectuel majeur du xIX ${ }^{\mathrm{e}}$ siècle breton.

Daniel PICHOT

Ghiati, Claude, Havelange, Isabelle, Keriven, Brigitte, Sonnet, Martine, Bibliographie annuelle de l'histoire de France du cinquième siècle à 1958. Année 2001, t. 47, CNRS éditions, Paris, 2002, 1080 p.

La Bibliographie annuelle de l'histoire de France qui rassemble chaque année depuis 1953 les articles et ouvrages français et étrangers concernant l'histoire de France a rendu un demi-siècle de bons et loyaux services à la communauté des historiens. Entre-temps, avec l'aide constante de la Bibliothèque (puis de la BNF), les ingénieures du CNRS responsables de l'ouvrage sont passées des fiches manuscrites à la saisie informatisée des données. Cette quarante-septième livraison de l'ouvrage rassemble 13085 notices provenant des dépouillements de 1287 revues françaises et 729 revues étrangères auxquels s'ajoutent 282 ouvrages collectifs (mélanges, actes de colloque, etc.). Le classement formel des références en neuf grandes rubriques qui reflète les principales directions de la recherche historique a bien entendu été conservé (" manuels généraux et sciences auxiliaires de l'histoire "; " histoire politique "; « histoire des institutions "; « histoire économique "; " histoire sociale "; " histoire religieuse "; " histoire de la France outre-mer "; " histoire de la civilisation "; « histoire locale "). 
Les conditions de préparation de l'ouvrage - alors même que les CNRS-éditions envisagent l'éventualité de la mise en ligne de ces données - n'ont pas permis la présentation des statistiques historiographiques habituelles. La reprise de l'" avertissement " liminaire dans lequel les auteurs commentent celles-ci est annoncée pour la prochaine livraison. On trouvera toutefois les tableaux publiés dans le tome 46 qui constituent un observatoire privilégié des oscillations de la recherche historique sur le site internet de l'Institut d'histoire moderne et contemporaine. Ce dernier comporte en effet une page consacrée à la $B A H F$ - http://www.ihmc.ens.fr/BAHF.html - dont la consultation est fort instructive. Pour ne tirer qu'une seule information des commentaires qu'appellent ces données, constatons que l'histoire économique, l'histoire sociale, l'histoire de l'enseignement, l'histoire des institutions, l'histoire religieuse, celle des sciences et celle du livre se distinguent par leur production abondante (articles français et étrangers confondus).

Quoi qu'il en soit de l'évolution prévisible du support de la $B A H F$ qui présenterait le double avantage subsidiaire d'être évolutif et ainsi mieux adapté à l'évolution des conditions de travail des historiens, l'affirmation sur ce site de la nécessité de poursuivre simultanément la publication des volumes imprimés est réconfortante. La pérennité de la version-papier de cet indispensable instrument de travail est gage de conservation à long terme et du confort de la consultation par les utilisateurs habituels. Bien entendu dans le présent volume comme dans les précédents, un triple index (index chronologique, index-matière et table des noms d'auteurs) met plus de trois cents pages au service du lecteur. Quelques améliorations ont toutefois été apportées. Dans l'index-matière, l'ordre alphabétique est désormais discontinu. De plus, le classement alphabétique des titres exclut enfin la prise en compte des articles, ce qui permet de se repérer plus facilement dans le cas des ouvrages collectifs (" Mélanges et Actes de congrès "). En consultant ce bel ouvrage, le plaisir de feuilleter demeure intact; s'y ajoute souvent pour le chercheur, celui de découvrir, par surcroît, ce qu'il ne s'attendait pas forcément à y trouver.

Bernard MERDRIGNAC 